\title{
Comparison between numerical-relativity and post-Newtonian waveforms from spinning binaries: the orbital hang-up case
}

\author{
Mark Hannam, ${ }^{1}$ Sascha Husa, ${ }^{2}$ Bernd Brügmann, ${ }^{1}$ and Achamveedu Gopakumar ${ }^{1}$ \\ ${ }^{1}$ Theoretical Physics Institute, University of Jena, 07743 Jena, Germany \\ ${ }^{2}$ Max-Planck-Institut für Gravitationsphysik, Albert-Einstein-Institut, Am Mühlenberg 1, 14476 Golm, Germany
}

(Dated: November 19, 2018)

\begin{abstract}
We compare results from numerical simulations of spinning binaries in the "orbital hangup" case, where the binary completes at least nine orbits before merger, with post-Newtonian results using the approximants TaylorT1, T4 and Et. We find that, over the ten cycles before the gravitational-wave frequency reaches $M \omega=0.1$, the accumulated phase disagreement between $\mathrm{NR}$ and $2.5 \mathrm{PN}$ results is less than three radians, and is less than 2.5 radians when using $3.5 \mathrm{PN}$ results. The amplitude disagreement between $\mathrm{NR}$ and restricted PN results increases with the black holes' spin, from about $6 \%$ in the equal-mass case to $12 \%$ when the black holes' spins are $S_{i} / M_{i}^{2}=0.85$. Finally, our results suggest that the merger waveform will play an important role in estimating the spin from such inspiral waveforms.
\end{abstract}

PACS numbers: 04.20.Ex, 04.25.Dm, 04.30.Db, 95.30.Sf

\section{INTRODUCTION}

Direct observation of gravitational waves may be at hand. Several ground-based gravitational-wave (GW) detectors have reached design sensitivity [1, 2, 3], and the recent completion of the LIGO S5 science run, where science data have been taken for almost two years, marks an important milestone in the field. With the joining of the GEO600 and Virgo detectors for part of this run, an important step has also been taken in establishing a global network of detectors, and data from all these detectors are currently being analyzed.

For black-hole binary systems, which are the strongest expected GW sources, current GW template banks use waveforms calculated by analytic post-Newtonian (PN) approximation methods to model waves from a binary's slow inspiral, and perturbation-theory techniques to model the ringdown of the final merged black hole. It is not clear how well these models describe the crucial merger phase, which will produce the strongest signal. Recent breakthroughs in numerical relativity (NR) [4, 5, 6] have made it possible to describe the late inspiral, merger and ringdown in full general relativity, and future GW searches will be able to use "hybrid" waveforms produced by combining PN and NR waveforms [7, 8, [9, 10].

The construction of hybrid waveforms requires an understanding of the frequency range in which $\mathrm{PN}$ and $\mathrm{NR}$ waveforms overlap, and how close to merger the PN waveforms cease to be sufficiently accurate. Studies so far have focused on nonspinning binaries $7,11,12,13,14$. The broad conclusion from that work was that up to a few orbits before merger, standard PN approximants predict the phase evolution of the binary to within an accuracy of about 1 radian [12, 13, 14], the lowest-order PN contribution to the wave amplitude disagrees with numerical results by about $6 \%$ over the frequency range considered in numerical simulations, and the error from higher-order PN amplitude corrections is reduced to only a few percent up to 10-15 orbits before merger 13, 14].

We now consider binaries in which the individual black holes are spinning. Spinning binaries have already been simulated in many contexts 15, 16, 17, 18, 19, 20, 21, $22,23,24,25,26,27,28,29,30,31]$, but in this work we present the first long (>15 GW cycles) simulations of spinning binaries, and compare with PN results during the inspiral. As a first example from the large parameter space, we consider equal-mass binaries with equal spins that are parallel to the orbital angular momentum of the binary. In addition, we compare the spinning waveforms with their nonspinning counterparts, and begin to address the question of how well the spin can be estimated from an observation of one of these waveforms. We find that, during the slow inspiral stage, spinning and nonspinning binaries are difficult to distinguish; while during the merger and ringdown the waveforms can be clearly distinguished.

We summarize our numerical methods in Section III, and also the main features of the numerical simulations that we performed, and in Section III summarize three PN calculations of a spinning binary's phase evolution, the Taylor T1, T4 and Et methods. In Section IV we compare the NR phase evolution and amplitude from the $(l=2, m=2)$ mode of $r \Psi_{4}$ with the PN phase calculated from the TaylorT1, T4 and Et approximants, and with the PN amplitude at restricted (quadrupole) order. We also compare the numerical waveforms with each other in Section $\nabla$ to get an estimate of how much nonspinning and spinning waveforms differ in the inspiral and merger phases.

\section{NUMERICAL METHODS AND RESULTS}

We performed numerical simulations with the BAM code [32, 33, 34]. The code starts with black-hole binary puncture initial data [35, 36] generated using a pseudo-spectral code [37], and evolves them with the $\chi$ - 
TABLE I: Summary of grid setup for numerical simulations. The grid parameters follow the notation introduced in 32]; see text. $h_{\min }$ denotes the resolution on the finest level and $h_{\max }$ the resolution on the coarsest level. The outer boundary of the computational domain is at approximately $r_{\max }$. The simulations with spin $S / M_{i}^{2}=0.75,0.85$ use one extra level of mesh refinement.

\begin{tabular}{l|r|r|r}
\hline \hline Run & $h_{\min }$ & $h_{\max }$ & $r_{\max }$ \\
\hline$S / M_{i}^{2}=0,0.25,0.5$ simulations & \\
\hline$\chi_{\eta=2}[5 \times 64: 5 \times 128: 6]$ & $M / 42.7$ & $12 M$ & $774 M$ \\
$\chi_{\eta=2}[5 \times 72: 5 \times 144: 6]$ & $M / 48.0$ & $32 / 3 M$ & $773 M$ \\
$\chi_{\eta=2}[5 \times 80: 5 \times 160: 6]$ & $M / 53.3$ & $48 / 5 M$ & $773 M$ \\
\hline$S / M_{i}^{2}=0.75,0.85$ simulations \\
\hline$\chi_{\eta=2}[6 \times 64: 5 \times 128: 6]$ & $M / 85.3$ & $12 M$ & $774 M$ \\
$\chi_{\eta=2}[6 \times 72: 5 \times 144: 6]$ & $M / 96.0$ & $32 / 3 M$ & $773 M$ \\
$\chi_{\eta=2}[6 \times 80: 5 \times 160: 6]$ & $M / 106.7$ & $48 / 5 M$ & $773 M$ \\
\hline \hline
\end{tabular}

variant of the moving-puncture [38, 39] version of the BSSN [40, 41] formulation of the 3+1 Einstein evolution equations. Spatial finite-difference derivatives are sixth-order accurate in the bulk [34], Kreiss-Oliger dissipation terms converge at fifth order, and a fourth-order Runge-Kutta algorithm is used for time evolution. The gravitational waves emitted by the binary are calculated from the Newman-Penrose scalar $\Psi_{4}$, and the details of our implementation of this procedure are given in 32].

The new simulations we performed for this analysis are summarized in Tables I and II All simulations are of equal-mass binaries; the black-hole punctures are placed on the $y$-axis at $y= \pm D / 2$, and given momenta in the $x$ direction of $p_{x}=\mp p$. The black holes each have the same spin angular momentum $S_{i}$ oriented parallel to the total orbital angular momentum, i.e., in the positive $z$ direction. The grid setup is described following the notation introduced in [32]. For example, $\chi_{\eta=2}[5 \times 64: 5 \times 128: 6]$ indicates that the simulation used the $\chi$ variant of the moving-puncture method, five nested mesh-refinement boxes with a base value of $64^{3}$ points surround each black hole, and five nested boxes with $128^{3}$ points surround the entire system, and there are six mesh-refinement buffer points. As summarized in Table II simulations were performed with spins $S / M_{i}^{2}=0.25,0.5,0.75,0.85$. We also make use of the results from nonspinning binaries, i.e., $S_{i} / M_{i}^{2}=0$, as reported in [13].

The physical parameters are given in Table II. As the spin is increased, the mass parameter $m_{i}$ decreases. This is partly because this quantity parametrizes the mass associated with the area of the apparent horizon, $M_{A H}=\sqrt{A / 16 \pi}$, where $A$ is the area of the horizon, while the total mass of the black hole is estimated by a variant of the Christodoulou formula [42],

$$
M_{i}^{2}=\left(M_{A H, i}\right)^{2}+\frac{S_{i}^{2}}{4\left(M_{A H, i}\right)^{2}} .
$$

If we are to keep $M_{i}$ constant as $S_{i}$ is increased, then $M_{A H}$ must decrease, and therefore $m_{i}$ decreases. The de-
TABLE II: Physical parameters for the moving-puncture simulations: the coordinate separation, $D / M$, the mass parameters in the puncture data construction, $m_{i} / M$, and the momenta $p_{x} / M$. The punctures are placed on the $y$-axis, and for all simulations the total initial black-hole mass is $M=1$.

\begin{tabular}{l|r|r|r|r}
\hline \hline Simulation & $S_{i} / M_{i}^{2}$ & $D / M$ & $m_{i} / M$ & $p_{x} / M$ \\
\hline S25 & 0.25 & 12.0 & 0.47579 & 0.083813 \\
S50 & 0.50 & 11.0 & 0.43277 & 0.087415 \\
S75 & 0.75 & 10.0 & 0.33608 & 0.091435 \\
S85 & 0.85 & 10.0 & 0.25628 & 0.090857 \\
\hline \hline
\end{tabular}

creasing value of $m_{i}$ is also due to the extra "junk" energy that the spin adds to the initial data: the Bowen-York extrinsic curvature used in the initial-data construction contains unwanted gravitational radiation that increases in amplitude as the spin is increased. That radiation adds to the mass of the black hole, and can be compensated by further lowering $m_{i}$, but only up to a point: eventually there is too much junk radiation, and we reach a limit in the $S_{i} / M_{i}^{2}$ that we can obtain. This limit has been found experimentally to be about $S_{i} / M_{i}^{2} \approx 0.928$ [43, 44]. A form of puncture data that permits higher spins has been suggested [45], but for the present study we consider spins no larger than $S_{i} / M_{i}^{2}=0.85$, which is well below the limit for Bowen-York data.

The initial momenta for quasi-circular inspiral were calculated using a 2.5-PN-accurate procedure based on the results in [46] and outlined in [28]. We expect these parameters to lead to inspiral with a small eccentricity, and this is indeed what we see in the numerical data; the eccentricity is typically of the order of $e \approx 0.006$. We found that the procedure described in [47] to produce lower-eccentricity inspiral, although applicable for spinning binaries, does not yet include sufficient accuracy in the spin terms to be of use for the scenarios described in this work.

We Richardson extrapolate our data with respect to numerical resolution and radiation extraction radius as described in 13. We first split the $(l=2, m=2)$ mode of the waveform $r \Psi_{4}$ into amplitude and phase according to

$$
r \Psi_{4}=A(\phi(t)) e^{-i \phi(t)},
$$

The amplitude is in turn written as a function of phase, $A(\phi)$, and this function is Richardson extrapolated with respect to numerical resolution. Five such functions are produced, one for each extraction radius, $R_{e x}=$ $\{50,60,70,80,90\} M$. We then note that the dependence of the amplitude on extraction radius is modeled well by

$$
A\left(\phi, R_{e x}\right)=A_{\infty}(\phi)+\frac{k(\phi)}{R_{e x}^{2}}+O\left(\frac{1}{R_{e x}^{3}}\right),
$$

and applying a curve fit of the form (3) to $A(\phi)$, we estimate $A\left(\phi, R_{e x} \rightarrow \infty\right)$. Although a method has been suggested to also extrapolate the phase to infinite extraction radius [14], in this work we again follow the proce- 
dure described in [13] and use the phase from the largest extraction radius, $R_{e x}=90 M$.

In the equal-mass case studied in [13], we found that the numerical results were cleanly sixth-order convergent, and were therefore able to both remove the sixth-order error term by Richardson extrapolation, and to estimate the uncertainty due to higher-order error terms. The present simulations of spinning binaries do not show such clean convergence. All of the simulations exhibit convergence between fifth and sixth order. Since the code contains fourth, fifth and sixth-order elements, it is not obvious at which resolutions each error term will dominate, and we make the most conservative choice of using the highest-resolution results and estimating an uncertainty by assuming only fourth-order convergence. Even with this conservative estimate of the discretization error, we find that, as in the equal-mass case, the errors are anyway dominated by the finite extraction radii. In general we estimate the uncertainty in our waveform amplitude as less than $3 \%$, and in the phase our uncertainty is 0.25 radians over the frequency range that we will consider for comparison with PN results.

These uncertainty estimates do not take into account the effect of eccentricity. In the nonspinning case studied in [13, 48], the eccentricity was $e<0.0016$, and the error in the phase evolution due to the eccentricity was estimated as being well below the finite-difference and finite-extractdion-radii errors. Figure 15 of [13] shows that the accumulated phase error of a simulation with $e \sim 0.008$ is around 0.2 radians, which is comparable to the numerical phase error. In the simulations presented in this work, the eccentricity can be as high at $e \sim 0.006$, and so significant eccentricity-induced phase errors may be expected. Based on Figure 15 in [13], we estimate that such errors are no larger than 0.2 radians. This is a systematic error: the binary merges sooner if the eccentricity is increased, and therefore the accumulated phase disagreement $\Delta \phi_{e}=\phi_{e=0}-\phi_{e>0}$ will always be positive. This systematic uncertainty should be taken into account in the comparisons we perform with PN approximants in Section IV] The effect of the eccentricity on the wave amplitude is to produce oscillations in the amplitude. For the simulations with higher spin, the eccentricity is larger, and the resulting oscillations are larger. This is clear in Figure 5] where the oscillations are visible in the highest-spin case shown $\left(S_{i} / M_{i}^{2}=0.75\right)$, and the highest-spin results are not shown because the oscillations reduce the clarity of the figure. We emphasize that the errors due to eccentricity may appear from our results to be larger for systems with larger spin, but this is not necessarily the case: the errors are simply larger when the eccentricity is large, and in the particular simulations we have done the larger-spin configurations also have larger eccentricity, and therefore larger eccentricityinduced errors.

Some of the features of the simulations are summarized in Table III] the mass and spin of the final black hole, and two quantities that allow comparison between simu-
TABLE III: Selected global features of the simulations: the mass and spin of the final black hole, $M_{\text {final }}$ and $S_{\text {final }} / M_{\text {final }}^{2}$; the time $\Delta t_{A}$ (in units of $M$ ) for the GW to evolve from $M \omega=0.06$ to its maximum amplitude; and the number of GW cycles $\Delta N_{G W}$ between $M \omega=0.06$ and the amplitude maximum.

\begin{tabular}{||l|r|r|r|r|}
\hline Simulation & $M_{\text {final }}$ & $S_{\text {final }} / M_{\text {final }}^{2}$ & $\Delta t_{A}(M)$ & $\Delta N_{G W}$ \\
\hline D12 & 0.950 & 0.680 & 719 & 11.0 \\
S25 & 0.943 & 0.757 & 819 & 12.7 \\
S50 & 0.932 & 0.826 & 917 & 14.7 \\
S75 & 0.920 & 0.896 & 1040 & 16.8 \\
S85 & 0.911 & 0.918 & 1096 & 18.0 \\
\hline
\end{tabular}

lations, and which demonstrate the orbital hangup effect first observed in numerical simulations in 15]. Starting from a GW frequency of $M \omega=0.06$, we list the time it takes the GWs to reach their maximum amplitude, $\Delta t_{A}$, and the number of GW cycles up to the maximum. (Dividing this number by two gives the corresponding number of the binary's orbits.) The numbers clearly show that the binary's merger is "hung up" by the presence of spin.

The mass of the final black hole was estimated by subtracting the radiated energy, as measured at the largest extraction radius $R_{e x}=90 M$, from the ADM mass of the spacetime, as calculated on the initial timeslice. The spin of the final black hole was estimated by comparing a fit of the ringdown with analytic quasinormal mode results. The values for the mass and spin are consistent with those already given in the literature [15, 22, 30].

Figure 1 shows the accumulated phase error between the nonspinning binary simulations presented in [13], and the various spin cases, for the ten cycles before the given spin waveform reaches a GW frequency of $M \omega=0.1$. The GW phases are aligned such that the phase difference is zero when $M \omega=0.1$ and we also relabel the time so that $t=0$ at this point; this procedure is described in more detail in Section IVA. We see that for $S_{i} / M_{i}^{2}=0.25$, the accumulated phase difference is about 1.6 radians, while for $S_{i} / M_{i}^{2}=0.85$, the accumulated phase difference is almost five radians. These differences should be borne in mind when we compare with the postNewtonian phase predictions.

\section{VARIOUS PRESCRIPTIONS FOR GW PHASE EVOLUTIONS IN PN RELATIVITY}

In this section, we provide formulae, extracted from Refs. [46, 49, 50, 51, 52, 53, 54, 55], required to construct TaylorT1, T4 and Et templates for inspiraling equal-mass binary black holes, having their spins $\boldsymbol{S}_{1}$ and $\boldsymbol{S}_{\mathbf{2}}$ aligned with the Newtonian angular momentum unit vector $\boldsymbol{l}$. (We retain $G$ and $c$ in the formulas in this section, although for the remainder of the paper we will adopt geometrized units $G=c=1$.) The aim is to provide $\mathrm{GW}$ phase evolutions under TaylorT1, T4 and Et prescriptions that include all the non-spinning contributions to 


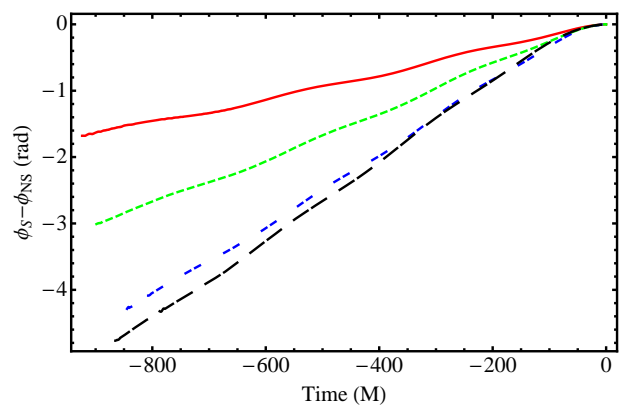

FIG. 1: The accumulated phase difference between nonspinning and spinning binaries for the ten cycles before $M \omega=0.1$, aligned such that the phase disagreement is zero when $M \omega=$ 0.1. The black-hole spins are, in order of increasing magnitude of the phase disagreement, $S_{i} / M_{i}^{2}=0.25,0.5,0.75,0.85$. Note that the differences are in some cases comparable to the phase disagreements with PN results that we will present in Section IV

$3.5 \mathrm{PN}$ order and the spin effects to $2.5 \mathrm{PN}$ order. We require, as in the case of non-spinning compact binaries, the dynamical (orbital) energy $\mathcal{E}(x)$, expressed as a PN series in terms of $x=\left(G M \omega_{b} / c^{3}\right)^{2 / 3}, \omega_{b}(t)$ being the binary's orbital angular frequency, and the GW energy luminosity $\mathcal{L}(x)$. The fact that we are dealing with compact binaries having aligned spins implies that to the highest PN order considered in this paper, the spin vectors $\boldsymbol{S}_{1}$ and $\boldsymbol{S}_{2}$ have their directions and magnitudes fixed.

The 3.5PN accurate $\mathcal{L}(x)$ and $3 \mathrm{PN}$ accurate $\mathcal{E}(x)$ associated with non-spinning comparable mass compact binaries are extractable from Refs. [49, 50, 51]. The lowestorder General Relativistic spin-orbit coupling, appearing at $1.5 \mathrm{PN}$ order for maximal Kerr black holes, and spinspin effects entering at $2 \mathrm{PN}$ order make contributions to $\mathcal{L}(x)$ and $\mathcal{E}(x)$ at relative $1.5 \mathrm{PN}$ and $2 \mathrm{PN}$ orders [46]. In the usual terminology adapted in the PN literature, this implies that spin-orbit coupling provides corrections to $\mathcal{E}(x)$ in terms of $x^{3 / 2}$ with respect to its Newtonian counterpart and a similar rule applies for $\mathcal{L}(x)$. The monopole-quadrupole interactions affect $\mathcal{L}(x)$ and $\mathcal{E}(x)$ at relative $2 \mathrm{PN}$ order [52]. The next to leading order General Relativistic spin-orbit coupling affects $\mathcal{L}(x)$ and $\mathcal{E}(x)$ at relative $2.5 \mathrm{PN}$ order $[54,55]$. However, for inspiraling spinning black holes, there exist corrections to $\mathcal{L}(x)$, also appearing at the relative $2.5 \mathrm{PN}$ order, due to the energy flowing in to the black holes. For comparable mass spinning black holes, the above-mentioned contributions are derived in Ref. [53]. For PN computations, available in Refs. [46, 449, 50, 51, 52, 54, 55], a non-spinning black hole is modeled as a point-particle (spherically symmetric mass distribution), and a spinning black hole is approximately treated as a spinning spherically symmetric mass distribution. It is important to note that properties of black holes, like presence of black hole horizons, play no role in these PN computations. Due to inclusion of black hole absorption effects, available in Ref. [53], it is reasonable to argue that our construction of fully $2.5 \mathrm{PN}$ accurate TaylorT1, T4 and Et templates are really applicable for spinning black hole binaries.

We define spins as $\boldsymbol{S}_{\boldsymbol{i}}=G m_{i}^{2} \chi_{i} \boldsymbol{s}_{\boldsymbol{i}}, i=1,2$ and in this paper, we impose the restrictions, $\boldsymbol{s}_{\boldsymbol{i}} \cdot \boldsymbol{l}=+1$ and $\boldsymbol{s}_{1} \cdot \boldsymbol{s}_{2}=+1$. For compact binaries having aligned spin configurations, it is also possible to employ the restricted $\mathrm{PN}$ waveforms

$$
h(t) \propto x(t)^{2 / 3} \cos 2 \phi(t),
$$

To obtain the GW phase evolution $\phi(t)$ in the TaylorT1 approximant, one numerically solves the following two differential equations:

$$
\begin{aligned}
\frac{d \phi(t)}{d t} \equiv \omega_{b}(t) & =\frac{c^{3}}{G M} x^{3 / 2}, \\
\frac{d x(t)}{d t} & =-\mathcal{L}(x)\left(\frac{d \mathcal{E}}{d x}\right)^{-1} .
\end{aligned}
$$

Therefore, to obtain the GW phase evolution, relevant for equal mass black hole binaries with aligned spins, under the TaylorT1 approximant that includes all the $3.5 \mathrm{PN}$ accurate non-spinning and $2.5 \mathrm{PN}$ accurate spin effects, we require the following expressions for $\mathcal{L}(x)$ and $\mathcal{E}(x)$.

$$
\begin{aligned}
\mathcal{L}(x) & =\left.\mathcal{L}(x)\right|_{\mathrm{NS}}+\left.\mathcal{L}(x)\right|_{\mathrm{S}}, \\
\left.\mathcal{L}(x)\right|_{\mathrm{NS}} & =\frac{2 c^{5}}{5 G} x^{5}\left\{1-\frac{373}{84} x+4 \pi x^{3 / 2}-\frac{59}{567} x^{2}\right. \\
& -\frac{767}{42} \pi x^{5 / 2}+\left[\frac{18608019757}{209563200}+\frac{355}{64} \pi^{2}-\frac{1712}{105} \gamma\right. \\
& \left.\left.-\frac{1712}{105} \ln (4 \sqrt{x})\right] x^{3}+\frac{16655}{6048} \pi x^{7 / 2}\right\}, \\
\left.\mathcal{L}(x)\right|_{\mathrm{S}} & =\frac{2 c^{5}}{5 G} x^{5}\left\{-\left(\chi_{1}+\chi_{2}\right) x^{3 / 2}+\frac{1}{2}\left(\chi_{1}{ }^{2}+\chi_{2}{ }^{2}\right.\right. \\
& \left.+\frac{31}{16} \chi_{1} \chi_{2}\right) x^{2}+\left[\frac{799}{504}\left(\chi_{1}+\chi_{2}\right)\right. \\
& \left.\left.-\frac{1}{32}\left(\chi_{1}+\chi_{2}+3 \chi_{1}{ }^{3}+3 \chi_{2}{ }^{3}\right) \nu\right] x^{5 / 2}\right\} \\
\mathcal{E}(x) & =\left.\mathcal{E}(x)\right|_{\mathrm{NS}}+\left.\mathcal{E}(x)\right|_{\mathrm{S}}, \\
\left.\mathcal{E}(x)\right|_{\mathrm{NS}} & =-\frac{M c^{2}}{8} x\left\{1+-\frac{37}{48} x-\frac{1069}{384} x^{2}\right. \\
& \left.+\left[\frac{1427365}{331776}-\frac{205}{384} \pi^{2}\right] x^{3}\right\}, \\
\left.\mathcal{E}(x)\right|_{\mathrm{S}} & =-\frac{M c^{2}}{8} x\left\{\frac{7}{6}\left(\chi_{1}+\chi_{2}\right) x^{3 / 2}-\frac{1}{4}\left(\chi_{1}+\chi_{2}\right)^{2} x^{2}\right. \\
& \left.+\frac{335}{144}\left(\chi_{1}+\chi_{2}\right) x^{5 / 2}\right\},
\end{aligned}
$$

where $\gamma$ is the Euler gamma, and where we use $\nu$ to mark contributions due to black-hole absorption effects; it is set to one to take those effects into account. 
The TaylorT4 approximant is obtained by Taylor expanding the right hand side of Eq. (5b) for $d x / d t$ and truncating it at the appropriate reactive $\mathrm{PN}$ order. Therefore, to construct GW phase evolution in the Taylor $\mathrm{T} 4$ approximant that contains all the $3.5 \mathrm{PN}$ accurate non-spinning and $2.5 \mathrm{PN}$ accurate spin effects, the following set of differential equations are numerically integrated:

$$
\begin{aligned}
& \frac{d \phi(t)}{d t} \equiv \omega_{b}(t)=\frac{c^{3}}{G M} x^{3 / 2}, \\
& \frac{d x(t)}{d t}=\left.\frac{d x(t)}{d t}\right|_{\mathrm{NS}}+\left.\frac{d x(t)}{d t}\right|_{\mathrm{S}}, \\
& \left.\frac{d x(t)}{d t}\right|_{\mathrm{NS}}=\frac{16 c^{3}}{5 G M} x^{5}\left\{1-\frac{487}{168} x+4 \pi x^{3 / 2}\right. \\
& +\frac{274229}{72576} x^{2}-\frac{254}{21} \pi x^{5 / 2} \\
& +\left[\frac{178384023737}{3353011200}-\frac{1712}{105} \gamma+\frac{1475}{192} \pi^{2}\right. \\
& \left.\left.-\frac{856}{105} \ln (16 x)\right] x^{3}+\frac{3310}{189} \pi x^{7 / 2}\right\} \text {, } \\
& \left.\frac{d x(t)}{d t}\right|_{\mathrm{S}}=\frac{16 c^{3}}{5 G M} x^{5}\left\{-\frac{47}{12}\left[\chi_{1}+\chi_{2}\right] x^{3 / 2}\right. \\
& +\left[\frac{5}{4}\left(\chi_{1}^{2}+\chi_{2}^{2}\right)+\frac{79}{32} \chi_{1} \chi_{2}\right] x^{2} \\
& +\left[-\frac{1}{32}\left(\chi_{1}+\chi_{2}+3 \chi_{1}{ }^{3}+3 \chi_{2}{ }^{3}\right) \nu\right. \\
& \left.\left.-\frac{8347}{2016}\left(\chi_{1}+\chi_{2}\right)\right] x^{5 / 2}\right\} \text {. }
\end{aligned}
$$

The construction of the TaylorEt approximant requires PN accurate expressions for $\omega_{b}$ in terms of $\mathcal{E}$. The radiation reaction induced inspiral is incorporated by expressing $\mathcal{L}$ in terms of $\mathcal{E}$. The restricted $\mathrm{PN}$ waveform associated with the TaylorEt approximant reads

$$
h(t) \propto \mathcal{E}(t) \cos 2 \phi(t),
$$

The PN accurate temporal evolutions for $\phi(t)$ and $\mathcal{E}(t)$, that includes all the $3.5 \mathrm{PN}$ accurate non-spinning and $2.5 \mathrm{PN}$ accurate spin effects, are obtained by solving the following coupled differential equations.

$$
\begin{aligned}
\frac{d \phi(t)}{d t} & \equiv \omega_{b}(t)=\left.\omega_{b}(t)\right|_{\mathrm{NS}}+\left.\omega_{b}(t)\right|_{\mathrm{S}} \\
\left.\omega_{b}(t)\right|_{\mathrm{NS}} & =\frac{c^{3}}{G M} \xi^{3 / 2}\left\{1+\frac{37}{32} \xi+\frac{12659}{2048} \xi^{2}\right. \\
& \left.+\left[\frac{205}{256} \pi^{2}+\frac{3016715}{196608}\right] \xi^{3}\right\} \\
\left.\omega_{b}(t)\right|_{\mathrm{S}} & =\frac{c^{3}}{G M} \xi^{3 / 2}\left\{-\frac{7}{4}\left[\chi_{1}+\chi_{2}\right] \xi^{3 / 2}\right. \\
& \left.+\frac{3}{8}\left[\left(\chi_{1}+\chi_{2}\right)^{2}\right] \xi^{2}-\frac{655}{64}\left[\chi_{1}+\chi_{2}\right] \xi^{5 / 2}\right\}
\end{aligned}
$$

$$
\begin{aligned}
\frac{d \xi(t)}{d t} & =\left.\frac{d \xi(t)}{d t}\right|_{\mathrm{NS}}+\left.\frac{d \xi(t)}{d t}\right|_{\mathrm{S}} \\
\left.\frac{d \xi(t)}{d t}\right|_{\mathrm{NS}} & =\frac{16 c^{3}}{5 G M} \xi^{5}\left\{1-\frac{197}{336} \xi+4 \pi \xi^{3 / 2}\right. \\
& +\frac{374615}{72576} \xi^{2}+\frac{299}{168} \pi \xi^{5 / 2}+\left[\frac{3155}{384} \pi^{2}\right. \\
& -\frac{1712}{105} \ln (4 \sqrt{\xi})+\frac{4324127729}{82790400} \\
& \left.\left.-\frac{1712}{105} \gamma\right] \xi^{3}+\frac{4155131}{96768} \pi \xi^{7 / 2}\right\} \\
\left.\frac{d \xi(t)}{d t}\right|_{\mathrm{S}} & =\frac{16 c^{3}}{5 G M} \xi^{5}\left\{-\frac{41}{6}\left[\chi_{1}+\chi_{2}\right] \xi^{3 / 2}\right. \\
& +\left[\frac{7}{4}\left(\chi_{1}^{2}+\chi_{2}^{2}\right)+\frac{111}{32} \chi_{1} \chi_{2}\right] \xi^{2} \\
& +\left[-\frac{1}{32}\left(\chi_{1}+\chi_{2}+3 \chi_{1}^{3}+3 \chi_{2}^{3}\right) \nu\right. \\
& \left.\left.-\frac{9943}{448}\left(\chi_{1}+\chi_{2}\right)\right] \xi^{5 / 2}\right\}
\end{aligned}
$$

where $\xi=-2 \mathcal{E} / \mu c^{2}$ and $\mu$ is the reduced mass. In this paper we keep $d \phi / d t$ to its highest $\mathrm{PN}$ order and change PN orders of $d \xi / d t$ to create various PN accurate TaylorEt approximants. The values of $\xi$ corresponding to any initial and final GW frequencies can be numerically evaluated using the right-hand side of Eq. (9a). This is possible due to the fact that for GWs from spinning compact binaries, having negligible orbital eccentricities, the frequency of the dominant harmonic is $f_{\mathrm{GW}} \equiv \omega_{b} / 2$.

We want to emphasize that the above mentioned Taylor approximants can only provide, from a strict $\mathrm{PN}$ point of view, fully $2.5 \mathrm{PN}$ accurate inspiral phase evolution for spinning black-hole binaries. This is because there are yet to be computed spin effects appearing at conservative and reactive $3 \mathrm{PN}$ and $3.5 \mathrm{PN}$ orders. Another point is that in these Taylor approximants, for the time being, we can only incorporate next-to-leading order spin-orbit effects and all other spin effects are mainly at the 'Newtonian order' (Newtonian order in the sense one refers to radiation reaction appearing for the first time at 2.5PN (absolute) order as Newtonian radiation reaction). Therefore, it is quite conceivable that smooth convergence to exact GW phase, reported in Ref. [48 for the TaylorEt approximant for non-spinning compact binaries, would not be present in its spinning counterpart.

\section{NR-PN COMPARISON DURING INSPIRAL}

In this section we compare the phase and amplitude of the numerical waveforms with that predicted by postNewtonian approximants. We have a lot of freedom in how to make such a comparison. Here we follow the procedure we used in [13], which is also used in [14]: we line up the phase and frequency of the PN and NR waveforms when the gravitational-wave frequency $M \omega$ is equal to 


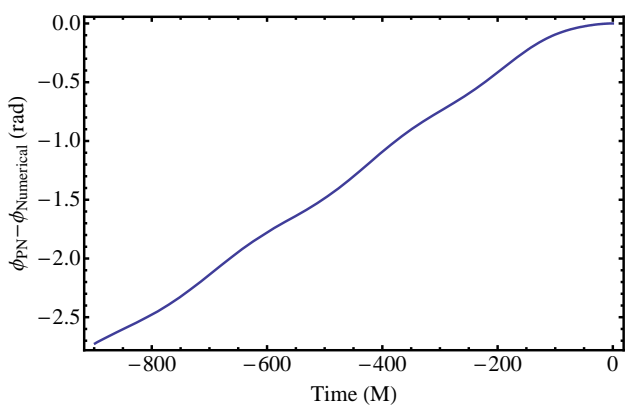

FIG. 2: An example of a phase comparison between NR and PN results. The case shown here is $S_{i} / M_{i}^{2}=0.5$, and the $\mathrm{PN}$ results were obtained with the TaylorT1 approximant at $2.5 \mathrm{PN}$ order. The accumulated phase error over the ten $\mathrm{GW}$ cycles before $M \omega=0.1$ is $\Delta \phi=-2.69$ radians.

0.1. This occurs several orbits before merger (the number of orbits depends on the value of the black-hole spin, as suggested by Table [II), at which time we expect postNewtonian results to still be valid. We then compare the phase and amplitude at earlier times. When comparing results between different cases, we need to decide whether to compare over a period of time, a frequency range, or a number of GW cycles. The results do not change qualitatively with different choices; we simply must make some choice; and the choice we make is to compare over a given number of GW cycles, which it seems to us will be most useful when later constructing hybrid waveforms. Our final procedure, then, is to compare the ten cycles before the GW frequency reaches $M \omega=0.1$. (This corresponds roughly to a black-hole orbital frequency of $M \omega_{b}=0.05$.)

\section{A. Phase}

As an example of how our comparison procedure works, Figure 2 shows the accumulated phase disagreement between the NR results for black holes with spins $S_{i} / M_{i}^{2}=0.5$, and the PN result using the Taylor $\mathrm{T} 1$ approximant at $2.5 \mathrm{PN}$ order. The phase disagreement is zero by construction when $M \omega=0.1$, and we choose $t=0$ at this point. As we progress backwards in time, the phase disagreement at first grows quadratically (since $\dot{\phi}_{P N}=\dot{\phi}_{N R}$ at $t=0,(\dot{\Delta \phi})=0$, and so there cannot be any linear growth in $\Delta \phi$ at "early" times). A linear dependence soon develops, however, and the phase disagreement grows roughly linearly for the remainder of the comparison, back to $t \approx-900 M$, when $\phi_{N R}$ has decreased by $20 \pi$, or ten GW cycles. Note also that there are wiggles in the plot; these are due to the small eccentricity in the binary that was simulated numerically, and we expect that they introduce an overall uncertainty in the numerical phase of about 0.2 radians with respect to a binary with zero eccentricity (see Figure 15 in [13]).

In the example shown in Figure 2 the accumulated phase error over the ten cycles before $M \omega=0.1$ is -2.69

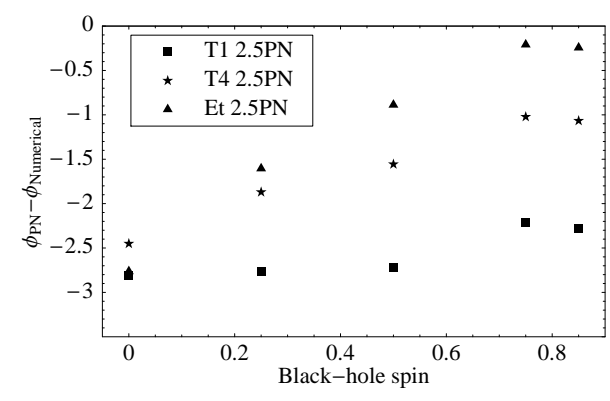

FIG. 3: The accumulated phase disagreement between NR and $\mathrm{PN}$ results over the ten cycles before $M \omega=0.1$. The five different spin values are $S_{i} / M_{i}^{2}=0,0.25,0.5,0.75,0.85$, and we compare with the three approximants TaylorT1, TaylorT4 and TaylorEt. All approximants are calculated at 2.5PN order.

radians. This is the most important piece of information we obtain from the plot, and rather than produce many very similar plots for different values of blackhole spin compared with different PN approximants at different PN orders, we summarize our results in Figures 3 and 4 . Here we see the accumulated phase agreement over ten cycles for five different values of spin, $\left(S_{i} / M_{i}^{2}=\{0,0.25,0.5,0.75,0.85\}\right)$, compared with three $\mathrm{PN}$ approximants, all calculated at 2.5PN and 3.5PN accuracy. The three approximants are the standard Taylor $\mathrm{T} 1$ approximant, the Taylor T4 approximant introduced in [14], and the Et approximant introduced in 56] and compared in detail with the nonspinning case in [48]. The small eccentricity tends to increase $\phi_{N R}$, so the points in Figures 3 and 4 are systematically too low by at most 0.2 radians for the spinning cases, and we should also recall the numerical uncertainty in $\phi_{N R}$ of 0.25 radians.

The main points we wish to emphasize in Figure 3 are that the accumulated phase disagreement between NR and 2.5PN TaylorT1 results is roughly constant for all values of black-hole spin. This suggests that when producing hybrid waveforms for these cases, the same number of numerical cyles are needed for all spin values to produce hybrid waveforms of the same phase accuracy. On the other hand, the T4 and Et approximants have the advantage that the phase disagreement is less than it is for T1, and decreases for higher spins, with the Et approximant performing best in the high-spin cases.

Figure 4 shows a similar plot, but this time the approximants are calculated up to $3.5 \mathrm{PN}$ accuracy. As pointed out in Section III, the 3.5PN results are not $3.5 \mathrm{PN}$-accurate in all terms, and these results will change when all 3.5PN terms are known and included.

We note once again that the phase disagreement between the NR and TaylorT1 phases is roughly constant for all values of spins. We also see that, as already seen in [14], the TaylorT4 approximant agrees extremely well with the NR phase in the nonspinning case. However, this does not hold for the spinning cases, and for larger spins 3.5PN TaylorT4 performs worse than TaylorT1. 


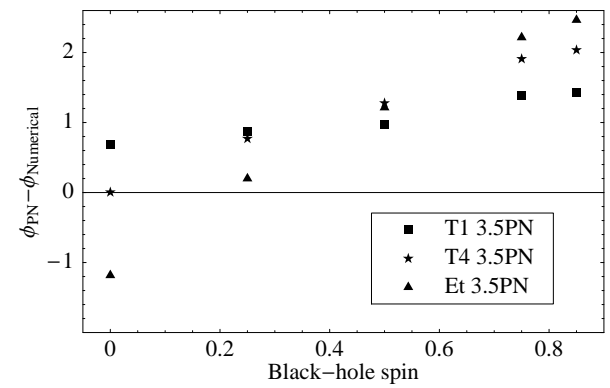

FIG. 4: The same comparison as in Figure 3, but this time the PN approximants are evaluated at 3.5PN order in those terms where this is possible (see text).

The new TaylorEt approximant agrees extremely well in one case (when $S_{i} / M_{i}^{2}=0.25$ ), but gives a large disagreement for high spins.

Our general conclusion from all of these comparisons is that, for the very small section of parameter space we have considered (equal-mass binaries with non-precessing spins in the "orbital hang-up" configuration), the phase agreement is roughly as good as in the nonspinning case; it certainly has not got dramatically worse, as one might have suspected. These numerical simulations can therefore be used to produce hybrid waveforms of comparable phase accuracy to those that can be produced from our previous nonspinning data $[8,9,13,57]$.

\section{B. Amplitude}

We now compare the amplitude of the $(l=2, m=$ 2) mode of the NR $r \Psi_{4}$ with that calculated from the restricted $\mathrm{PN}$ approximation, which is the same as the amplitude from the quadrupole formula. The restricted PN amplitude of the GW strain $h$ is given by $A_{\text {restricted }}=$ $(M \omega / 2)^{2 / 3} / R$, where $R$ is the distance from the source, and we have

$$
h=A_{\text {restricted }} e^{i \phi_{P N}(t)} .
$$

The phase $\phi_{P N}(t)$ depends on the PN approximant used in the previous section. However, if we differentiate $h$ twice with respect to time to calculate $r \Psi_{4,22}$, and consider the amplitude as a function of GW frequency $M \omega$, then we find that the choice of approximant has a negligible effect on the resulting function. In other words, the GW strain amplitude $A_{\text {restricted }}$ depends only on the GW frequency, and so does the restricted PN amplitude of $r \Psi_{4,22}$, to a good approximation. This point was also made in [14]. This fact allows us to make a comparison between the NR and restricted PN amplitudes that is independent of the PN approximant that we used to calculate the phase - the results are independent of whether we use Taylor T1, T4, or Et.

In the nonspinning case we found that the NR and restricted PN amplitudes disagree by about $(6 \pm 2) \%[13$,

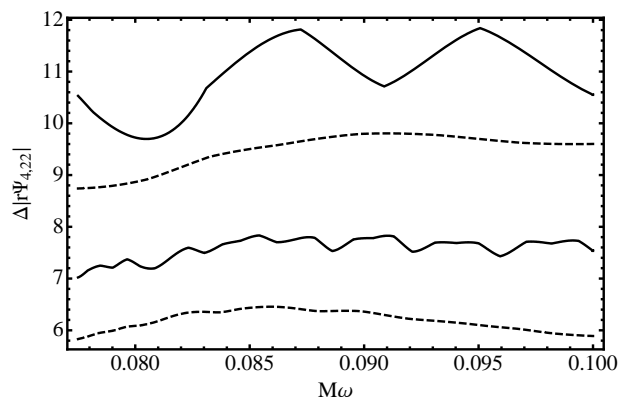

FIG. 5: The disagreement between restricted $\mathrm{NR}$ and $\mathrm{PN}$ amplitudes of $r \Psi_{4,22}$ as a function of GW frequency $M \omega$. The lines below correspond, from bottom to top, to the cases $S_{i} / M_{i}^{2}=0,0.25,0.5,0.75$. (Every second line is dashed, to make them easier to distinguish.) In the nonspinning case the disagreement is roughly $6 \%$ over this frequency range. The disagreement increases as spin is added, and is about $11 \%$ for $S_{i} / M_{i}^{2}=0.75$. The large oscillations in this last case are due to the relatively high eccentricity of that system, $e \sim 0.006$.

while 14 found $5 \%$ disagreement using numerical waveforms with higher accuracy. Both sets of numerical results found that the disagreement was roughly constant over the last ten cycles before $M \omega=0.1$.

The results for the spinning binaries we have considered are shown in Figure 5 , We see that for spinning binaries the amplitude disagreement increases. The plot shows results for spins $S_{i} / M_{i}^{2}=0,0.25,0.5,0.75$. For the highest spin shown here, the amplitude disagreement between NR and restricted PN is about $11 \%$. In the case of $S_{i} / M_{i}^{2}=0.85$, fluctuations due to eccentricity make it difficult to clearly measure the amplitude disagreement, and that case is not shown in the plot; however, we estimate the disagreement at around $12 \%$, which is twice as high as in the nonspinning case.

Higher-order PN amplitude corrections were able to improve the agreement in the nonspinning case to around $2 \%$ using 2.5PN amplitude corrections [13, 14], and even better agreement was found when using $3 \mathrm{PN}$ amplitude corrections [14]. It is clear from our current results that higher-oder PN amplitude corrections are even more crucial when spins are included. Higher-order corrections up to $2.5 \mathrm{PN}$ already exist [46, 54, 55], but 3PN amplitudes are not yet known for spinning binaries. These corrections may substantially improve the agreement with NR amplitudes, but we will postpone that study to future work.

\section{INSPIRAL AND MERGER}

We may ask how much the waveforms from spinning and nonspinning binaries differ. In data analysis applications the overlap integrals between different waveforms determine how well a GW search will distinguish between them. If the phase of two waveforms can be lined up such that the waveforms are identical, then the overlap 
between them is one. If the waveforms differ slightly, the value of the overlap integral falls below one. In real-world data-analysis applications, the detector noise is included in the overlap integral, which leads to results that depend on the total mass of the binary. For the purposes of the purely qualitative illustration in this section, we do not use the detector noise, and we calculate the overlap integrals in the time domain. Given some part of the waveform, we may calculate an overlap integral of the form,

$$
O(\delta t, \delta \phi)=\frac{1}{N_{N S} N_{S}} \int_{t_{1}}^{t_{2}} h_{N S}(t) h_{S}^{\star}(t+\delta t, \delta \phi) d t,
$$

where "NS" indicates the waveform from a nonspinning binary, and "S" indicates one of the spinning-binary waveforms. The spinning waveform is time-shifted by some $\delta t$, and phase-shifted by some $\delta \phi$. The normalization factors are calculated by performing the integrals

$$
\begin{aligned}
N_{N S}^{2} & =\int_{t_{1}}^{t_{2}}\left|h_{N S}(t)\right|^{2} d t, \\
N_{S}^{2} & =\int_{t_{1}}^{t_{2}}\left|h_{S}(t)\right|^{2} d t .
\end{aligned}
$$

For some choice of $t_{1}$ and $t_{2}$, the time and phase shifts $\delta t$ and $\delta \phi$ can be optimized to find the largest possible overlap.

Let us compare the nonspinning-binary waveform with that from a binary with $S_{i} / M_{i}^{2}=0.75$. We first choose a time interval that includes only inspiral, $t_{1}=1200 \mathrm{M}$ and $t_{2}=1700 \mathrm{M}$ with respect to the nonspinning waveform; this corresponds to roughly five GW cycles in the frequency range $M \omega \approx 0.06$ to $M \omega \approx 0.09$. The maximum overlap that can be achieved when comparing this small number of cycles with the S75 simulation is just under 0.99 , meaning that the overlap is extremely good. This illustrates that during the inspiral, it is difficult to distinguish between the spinning and nonspinning waveforms. Put another way, if one of these waveforms were detected, a large number of cycles would be required to estimate the black holes' spins. In practice, indeed many hundreds or thousands of cycles may be detected during the inspiral, and the accumulated phase difference between spinning and nonspinning binaries may be more obvious. However, at present we must use PN approximants to model those hundreds or thousands of cycles, and it is not entirely clear how the variation in number of cycles when spin is added compares with the deviation of the PN approximants at different PN orders from the true solution.

In addition, if a noticeable phase difference between spinning and nonspinning waveforms does accumulate over many hundreds of cycles, this might be partially compensated by rescaling the total mass of one of the binaries; a spinning binary may thus be detected as a nonspinning binary with the wrong mass.

We now examine a time interval that includes the merger, $t_{1}=1870 M$ and $t_{2}=2020 M$. The maximum
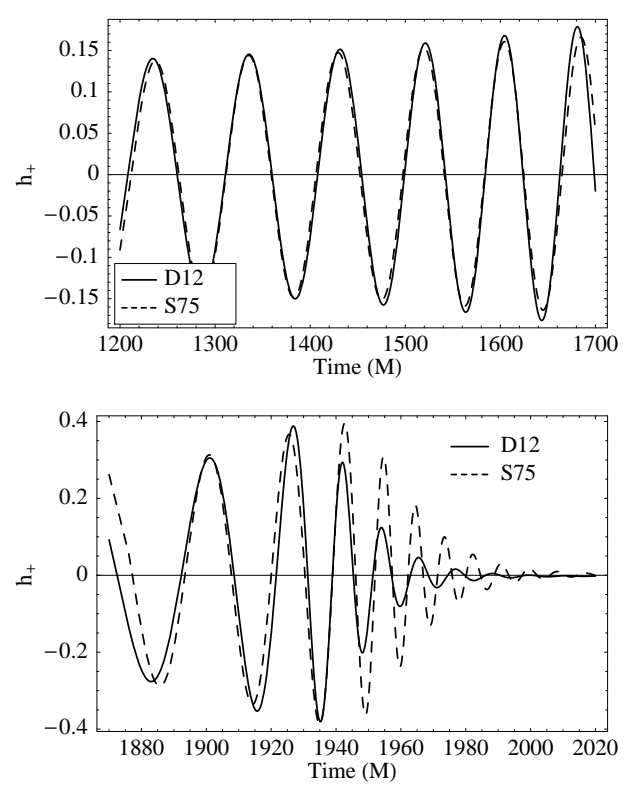

FIG. 6: Spinning and nonspinning waveforms in the time intervals $t=[1200,1700]$ and $t=[1870,2020]$, with the time and phase shifted so that they give the maximum value of the overlap integral (11). We can clearly see by eye that the overlap for the earlier time interval is much larger than that for the later interval.

overlap that we can now achieve is less than 0.90: the waveforms differ far more in the merger phase. This is a far greater difference than we observe during the inspiral, and a far greater difference than we would expect to see even if many more inspiral cycles were available.

Furthermore, if we were to adjust the total mass of one of the binaries, such that the overlap of the inspiral waveforms was increased to almost one, this would not significantly improve the overlap of the merger waveforms. Therefore, detecting both the inspiral and merger may allow a significantly better estimate of the black holes' parameters.

In Figure 6 we illustrate this point by showing the waveforms for the nonspinning and $S_{i} / M_{i}^{2}=0.75$ simulations, over the two time intervals we just discussed, and lined up such that the overlap integral (11) is a maximum. It is immediately clear from the two plots that the overlap will be larger for the earlier time interval.

We conclude from this simple analysis that, at least for binaries in an orbital hangup configuration, an accurate estimate of the black holes' spins will be far easier if the merger waveform is detected, rather than the inspiral. And, perhaps not surprisingly, the most accurate parameter estimation would be possible if both the inspiral and merger were detected. A similar conclusion can be made when looking at higher modes, although all modes up to $l=4$ have an amplitude no more than $10 \%$ that of the $(l=2, m=2)$ mode in the cases we consider. 


\section{DISCUSSION}

We have extended our previous comparison of NR and PN inspiral waveforms [13] to spinning binaries. In particular, we considered equal-mass binaries whose black holes have equal spins oriented parallel to the orbital angular momentum. In these cases the spins do not precess and there is no net radiated linear momentum, but there is an "orbital hangup" effect that delays the merger as the spin is increased [15].

We compared the accumulated phase difference between NR and PN approximants over the ten cycles before the $\mathrm{GW}$ frequency reaches $M \omega=0.1$. We used the PN approximants TaylorT1, T4 and Et, at orders 2.5PN and 3.5PN. We conclude that, as in the nonspinning case, the growth of phase error is fairly small; for the number of cycles we considered, it is less than 2.5 radians for all 3.5PN approximants for spins up to $S_{i} / M_{i}^{2}=0.85$, and less than 1.5 radians for the TaylorT1 approximant. The 3.5PN TaylorT4 approximant, which performed extremely well in the equal-mass case [14] is seen to perform much worse in the spinning cases. However, we stress that not all terms in the PN approximants are known to $3.5 \mathrm{PN}$ order. At $2.5 \mathrm{PN}$ order (the highest at which all terms are known), the Et approximant gives the best phase agreement. These results are summarized in Figures 3 and 4.

We also compare the amplitude of the $(l=2, m=2)$ mode of the NR $r \Psi_{4}$ with that calculated by the restricted PN approximation. In the equal-mass case the PN amplitude was larger than its NR counterpart by about $6 \%$. In a binary whose black holes have spins $S_{i} / M_{i}^{2}=0.85$, the amplitude disagreement grows to around $12 \%$.

Finally, we gave an illustration of how the inspiral and merger waveforms differ between the spinning and nonspinning cases. Our simple analysis suggests that the spin is difficult to distinguish during inspiral, but has an extremely clear effect on the merger waveform. As such, we expect that estimating the spins of a binary's constituents should be much easier in cases where the merger is also detected.

In the future we intend to extend this analysis to a larger sample of parameter space (in particular, more general spin configurations), and to explore the dataanalysis implications of our results.

\section{Acknowledgments}

AG is grateful to Gerhard Schäfer for fruitful discussions and Manuel Tessmer for crosschecking PN formulae, and $\mathrm{SH}$ and $\mathrm{MH}$ for discussions with Alicia Sintes. This work was supported in part by DFG grant SFB/Transregio 7 "Gravitational Wave Astronomy" and the DLR (Deutsches Zentrum für Luft- und Raumfahrt) through "LISA Germany". We thank the DEISA Consortium (co-funded by the EU, FP6 project 508830), for support within the DEISA Extreme Computing Initiative (www.deisa.org). Computations were performed at LRZ Munich and the Doppler and Kepler clusters at the Institute of Theoretical Physics of the University of Jena.
[1] S. Waldman (for the LIGO Scientific Collaboration), Class. Quantum Grav. 23 (2006) S653 - S660 (2006).

[2] S. Hild (for the LIGO Scientific Collaboration), Class. Quantum Grav. 23, S643 (2006).

[3] F. Acernese et al., Class. Quantum Grav. 23, S635 (2006).

[4] F. Pretorius, Phys. Rev. Lett. 95, 121101 (2005), grqc/0507014.

[5] M. Campanelli, C. O. Lousto, P. Marronetti, and Y. Zlochower, Phys. Rev. Lett. 96, 111101 (2006), grqc/0511048.

[6] J. G. Baker, J. Centrella, D.-I. Choi, M. Koppitz, and J. van Meter, Phys. Rev. Lett. 96, 111102 (2006), grqc/0511103.

[7] Y. Pan et al., Phys. Rev. D77, 024014 (2008), 0704.1964.

[8] P. Ajith, S. Babak, Y. Chen, M. Hewitson, B. Krishnan, J. T. Whelan, B. Brügmann, P. Diener, J. González, M. Hannam, et al., Class. Quantum Grav. 24, S689 (2007), arXiv:0704.3764 [gr-qc].

[9] P. Ajith et al., Phys. Rev. D77, 104017 (2008), 0710.2335.

[10] A. Buonanno et al., Phys. Rev. D76, 104049 (2007), 0706.3732.

[11] A. Buonanno, G. B. Cook, and F. Pretorius, Phys. Rev. D75, 124018 (2007), gr-qc/0610122.
[12] J. G. Baker, J. R. van Meter, S. T. McWilliams, J. Centrella, and B. J. Kelly, Phys. Rev. Lett. 99, 181101 (2007), gr-qc/0612024.

[13] M. Hannam, S. Husa, J. A. González, U. Sperhake, and B. Brügmann, Phys. Rev. D 77, 044020 (2008), arXiv:0706.1305 [gr-qc].

[14] M. Boyle et al., Phys. Rev. D76, 124038 (2007), 0710.0158

[15] M. Campanelli, C. O. Lousto, and Y. Zlochower, Phys. Rev. D 74, 041501(R) (2006), gr-qc/0604012.

[16] M. Campanelli, C. O. Lousto, Y. Zlochower, B. Krishnan, and D. Merritt, Phys. Rev. D75, 064030 (2007), gr-qc/0612076.

[17] M. Campanelli, C. O. Lousto, Y. Zlochower, and D. Merritt, Astrophys. J. 659, L5 (2007), gr-qc/0701164.

[18] M. Campanelli, C. O. Lousto, Y. Zlochower, and D. Merritt, Phys. Rev. Lett. 98, 231102 (2007), gr-qc/0702133.

[19] C. O. Lousto and Y. Zlochower (2007), arXiv:0708.4048 [gr-qc].

[20] M. Koppitz, D. Pollney, C. Reisswig, L. Rezzolla, J. Thornburg, P. Diener, and E. Schnetter, Phys. Rev. Lett. 99, 041102 (2007).

[21] D. Pollney et al., Phys. Rev. D76, 124002 (2007), 0707.2559 .

[22] L. Rezzolla et al., Astrpphys. J679, 1422 (2008), 
0708.3999.

[23] L. Rezzolla et al. (2007), arXiv:0710.3345 [gr-qc].

[24] F. Herrmann, I. Hinder, D. Shoemaker, P. Laguna, and R. A. Matzner (2007), gr-qc/0701143.

[25] F. Herrmann, I. Hinder, D. M. Shoemaker, P. Laguna, and R. A. Matzner, Phys. Rev. D76, 084032 (2007), 0706.2541.

[26] J. G. Baker et al. (2007), astro-ph/0702390.

[27] J. A. González, M. D. Hannam, U. Sperhake, B. Brügmann, and S. Husa, Phys. Rev. Lett. 98, 231101 (2007), gr-qc/0702052.

[28] B. Brügmann, J. A. González, M. Hannam, S. Husa, and U. Sperhake (2007), arXiv:0707.0135 [gr-qc].

[29] E. Berti, V. Cardoso, J. A. Gonzalez, U. Sperhake, and B. Bruegmann (2007), arXiv:0711.1097 [gr-qc].

[30] P. Marronetti, W. Tichy, B. Brügmann, J. González, and U. Sperhake, Phys. Rev. D 77, 064010 (2008), arXiv:0709.2160 [gr-qc].

[31] W. Tichy and P. Marronetti (2007), gr-qc/0703075.

[32] B. Brügmann, J. A. González, M. Hannam, S. Husa, U. Sperhake, and W. Tichy, Phys. Rev. D 77, 024027 (2008), gr-qc/0610128.

[33] B. Brügmann, W. Tichy, and N. Jansen, Phys. Rev. Lett. 92, 211101 (2004), gr-qc/0312112.

[34] S. Husa, J. A. González, M. Hannam, B. Brügmann, and U. Sperhake, Class. Quantum Grav. 25, 105006 (2008), arXiv:0706.0740 [gr-qc].

[35] S. Brandt and B. Brügmann, Phys. Rev. Lett. 78, 3606 (1997), gr-qc/9703066.

[36] J. M. Bowen and J. W. York, Phys. Rev. D 21, 2047 (1980).

[37] M. Ansorg, B. Brügmann, and W. Tichy, Phys. Rev. D 70, 064011 (2004), gr-qc/0404056.

[38] M. Campanelli, C. O. Lousto, P. Marronetti, and Y. Zlochower, Phys. Rev. Lett. 96, 111101 (2006), grqc/0511048.

[39] J. G. Baker, J. Centrella, D.-I. Choi, M. Koppitz, and J. van Meter, Phys. Rev. Lett. 96, 111102 (2006), gr- qc/0511103

[40] M. Shibata and T. Nakamura, Phys. Rev. D 52, 5428 (1995).

[41] T. W. Baumgarte and S. L. Shapiro, Phys. Rev. D 59, 024007 (1998), gr-qc/9810065.

[42] D. Christodoulou, Phys. Rev. Lett. 25, 1596 (1970).

[43] J. W. York, Jr. and T. Piran, in Spacetime and Geometry: The Alfred Schild Lectures, edited by R. A. Matzner and L. C. Shepley (University of Texas Press, Austin (Texas), 1982), pp. 147-176, ISBN 0-292-77567-9.

[44] M. W. Choptuik and W. G. Unruh, Gen. Rel. Grav. 18, 813 (1986).

[45] M. Hannam, S. Husa, B. Brügmann, J. A. Gonzalez, and U. Sperhake, Class. Quantum Grav. 24, S15 (2007), arXiv:gr-qc/0612001.

[46] L. E. Kidder, Phys. Rev. D 52, 821 (1995).

[47] S. Husa, M. Hannam, J. A. González, U. Sperhake, and B. Brügmann, Phys. Rev. D (2007), arXiv:0706.0904 [gr$\mathrm{qc}]$.

[48] A. Gopakumar, M. Hannam, S. Husa, and B. Brügmann (2007), arXiv:0712.3737 [gr-qc].

[49] T. Damour, P. Jaranowski, and G. Schäfer, Phys. Lett. B 513, 147 (2001), gr-qc/0105038.

[50] L. Blanchet, G. Faye, B. R. Iyer, and B. Joguet, Phys. Rev. D 65, 061501 (2002), erratum Phys. Rev. D71, 129902 (2005)., gr-qc/0105099.

[51] L. Blanchet, T. Damour, G. Esposito-Farese, and B. R. Iyer, Phys. Rev. Lett. 93, 091101 (2004), gr-qc/0406012.

[52] E. Poisson, Phys. Rev. D57, 5287 (1998), gr-qc/9709032.

[53] K. Alvi, Phys. Rev. D64, 104020 (2001), gr-qc/0107080.

[54] L. Blanchet, A. Buonanno, and G. Faye, Phys. Rev. D74, 104034 (2006), gr-qc/0605140.

[55] G. Faye, L. Blanchet, and A. Buonanno, Phys. Rev. D74, 104033 (2006), gr-qc/0605139.

[56] A. Gopakumar (2007), arXiv:0712.3236 [gr-qc].

[57] P. Ajith (2007), arXiv:0712.0343 [gr-qc]. 